\title{
The search for treatments to reduce chemotherapy-induced peripheral neuropathy
}

\author{
Deirdre R. Pachman, ${ }^{1}$ Charles L. Loprinzi, ${ }^{1}$ Axel Grothey, ${ }^{1}$ and Lauren E. Ta ${ }^{2}$
}

1Division of Medical Oncology and 2Department of Neurology, Mayo Clinic, College of Medicine, Rochester, Minnesota, USA.

\begin{abstract}
Oxaliplatin, a commonly used chemotherapeutic agent, is associated with both acute and chronic neurotoxicity. Chronic sensory neuropathy can be dose limiting and may have detrimental effects on patients' quality of life. Preclinical studies provide an understanding of the pathophysiology of chemotherapy-induced peripheral neuropathy (CIPN) and may be important for developing effective preventative interventions. In this issue of the JCI, Coriat and colleagues used an animal model and a human pilot trial to evaluate the use of mangafodipir to reduce CIPN. Although many pilot clinical studies have reported promising data, larger clinical trials have repeatedly been unable to confirm these preliminary results. Thus, no agents are currently clinically recommended for the prevention of CIPN.
\end{abstract}

\section{Oxaliplatin-associated neuropathy is a substantial problem}

Oxaliplatin is a commonly used platinum-based chemotherapeutic agent that frequently needs to be stopped due to neurotoxicity. The development of oxaliplatin-associated neurological symptoms can substantially affect patients' quality of life and functional ability, and these neurologic defects can last for years in some patients (1). The benefits of this drug are constantly being weighed against the risk of permanent neurologic disorder. Consequently, there has been extensive research into methods to prevent this troublesome toxicity.

\section{Animal data indicate that drugs can prevent CIPN}

A better understanding of the pathophysiology and molecular mechanisms responsible for chemotherapy-induced peripheral neuropathy (CIPN) is important for the development of effective preventative interventions. Emerging evidence suggests that alterations in the expression or activity of antioxidants, such as glutathione reductase, catalase, and superoxide dismutases (SODs), increase the susceptibility of neurons to ROS-mediated injury, which contributes to neurotoxicity (2). Preclinical studies have shown that the platinum-based chemotherapeutic drug cisplatin generates ROS in dorsal root

Conflict of interest: The authors have declared that no conflict of interest exists.

Citation for this article: $J$ Clin Invest. 2014; 124(1):72-74. doi:10.1172/JCI73908. ganglia (DRG) neurons (3). NO, as well as other inflammatory byproducts, is capable of directly activating neuronal transient receptor potential channel A1 (TRPA1) (4), which has been shown to be upregulated in DRG and the trigeminal ganglion in vitro and in vivo following platinum drug treatment $(5,6)$. In addition, mitochondrial damage induced by oxidative stress has been suggested as a mechanism involved in neurotoxicity following oxaliplatin treatment (7). Recently, it has also been proposed that activation of poly(ADP-ribose) polymerase (PARP) contributes to neuroinflammation and increased oxidative stress (8).

These studies of the pathophysiology and molecular mechanisms of CIPN have led to animal studies of agents that might be able to prevent CIPN. Research in animal models of CIPN has suggested that a variety of agents can decrease neurological symptoms. Drugs demonstrating promise in animal models include PARP inhibi(10), glutathione (11), erythropoietin (12), and goshajinkigan (a Japanese traditional herbal medicine) (13).

In this issue of the JCI, Romain Coriat and colleagues provide support that mangafodipir can be added to the list of drugs that relieve CIPN in animals (14). Coriat et al. demonstrate that mice treated with oxaliplatin and the MRI contrast agent mangafodipir, or oxaliplatin and MnTBAP, which is a manganese chelate with SOD and catalase activities, did not develop mechanical hypersensitivity, cold hypersensitivity, or tors (8), acetyl-L-carnitine (9), minocycline deficits in motor function (14). The results of mouse pain behavioral studies are fairly straightforward; however, the neurotoxic effects of oxaliplatin on myelinated fibers of the sciatic nerve will require further study. Coriat et al. report a decrease in myelinated fiber diameter, but no change in axon diameter following oxaliplatin treatment (14). These results would suggest that oxaliplatin reduces myelin thickness; however, myelin is not typically affected by oxaliplatin (15). Morphometric and histological studies of nerve fibers will be required to determine whether mangafodipir is neuroprotective or myelin protective.

\section{CIPN-preventing drugs implicated in pilot studies lack benefit in larger trials}

Vitamin E was one of the first compounds thought to protect against CIPN. Data supporting vitamin $\mathrm{E}$ for the treatment of CIPN (16-18) came from three small randomized trials with unblinded control groups (19-21) and one larger trial that included 17 patients treated with vitamin E (22). Unfortunately, a much larger randomized, placebo-controlled, double-blind clinical trial was unable to support the use of vitamin $\mathrm{E}$ for CIPN treatment or prevention (23).

The use of i.v. calcium and magnesium $(\mathrm{Ca} / \mathrm{Mg})$ for CIPN prevention became a common clinical practice after a report that compared a series of patients treated with i.v. $\mathrm{Ca} / \mathrm{Mg}$ with a historical control group suggested that i.v. $\mathrm{Ca} / \mathrm{Mg}$ decreased neuropathy by about $50 \%$ (24). Furthermore, data from a placebo-controlled, double-blind clinical trial suggested that i.v. $\mathrm{Ca} / \mathrm{Mg}$ was beneficial (25); however, this trial was halted due to the errant suggestion that $\mathrm{Ca} / \mathrm{Mg}$ interfered with the response rate of oxaliplatin-based chemotherapy $(26,27)$. As with vitamin $E$, a large phase III clinical trial on the use of i.v. Ca/Mg for preventing CIPN determined that this treatment was ineffective (28).

In 1990, a report in the New England Journal of Medicine indicated that an adrenocorticotropic hormone analog (ORG 2766) 
relieved CIPN. A total of 55 patients were involved in a three-arm study that included a placebo, a low dose of ORG 2766, and a higher dose of ORG 2766 (29). The trial authors reported a substantial improvement in neuropathy, suggesting that this therapy was effective in preventing or attenuating cisplatin neuropathy (29). A follow-up report indicated that a small subset of 18 patients from the previous study had less pronounced neurologic signs and symptoms months after finishing their chemotherapy; this report recommended that ORG 2766 be continued for up to 4 months after the last cycle of cisplatin (30). Follow-up studies by some of the same authors further reported that ORG 2766 relieved nerve damage (31). Another small trial involving 28 patients who were receiving vincristine also reported positive results (32). Despite the initially promising studies, two relatively large, well-conducted, placebo-controlled clinical trials could not correlate the use of ORG 2766 with decreased neuropathy $(33,34)$. Moreover, in one trial, ORG 2766 was associated with increased neuropathy (34).

Multiple small trials have reported positive results using glutathione for the prevention of cisplatin- or oxaliplatin-related CIPN (35-37). No large, definitive phase III trials have been reported to confirm or refute the ability of glutathione to prevent oxaliplatin- or cisplatin-induced CIPN; however, a relatively large randomized, placebo-controlled, double-blind trial failed to find a glutathione-associated benefit for preventing the neuropathy associated with paclitaxel/carboplatin (C.L. Loprinzi, unpublished observations).

Additionally, a small phase II study suggested that acetyl-L-carnitine could improve chemotherapy-induced neuropathic symptoms (38). Based on this study, a phase III clinical prevention trial was conducted in patients receiving paclitaxel. In this trial, acetyl-L-carnitine appeared to be associated with increased chemotherapy-induced neuropathy (39).

In the current report, Coriat et al. provide results from a phase II clinical trial using mangafodipir in patients with preexisting oxaliplatin-induced CIPN (14). The trial involved 22 patients with at least grade 2 sensory neuropathy. After 4 cycles of oxaliplatin and mangafodipir, they reported that 17 patients had stable or improved neuropathy, and after 8 cycles, 6 patients had improvement in their neuropathy grade. As oxaliplatin-induced CIPN is expected to worsen with cumulative doses, these findings do sound intriguing. Unfortunately, to date, none of the previously reported promising-appearing pilot studies have shown clinical benefit when tested in large randomized clinical trials. Thus, more work will need to be done to determine whether mangafodipir will really benefit patients with CIPN.

\section{Perspectives and future directions}

The development of CIPN is a pertinent clinical problem that needs to be addressed. It is well established that oxaliplatinmediated neurotoxicity correlates with a cumulative oxaliplatin dose; therefore, International Duration Evaluation in the Adjuvant colon cancer (IDEA) trial, an international collaborative clinical trial, is underway to evaluate whether 3 months of oxaliplatin treatment provide the same benefit as the current standard of 6 months of adjuvant oxaliplatin-based therapy (40). This effort will eventually include about 12,000 patients worldwide and could have major implications for the long-term quality of life and functional capabilities of patients with resected colon cancer. Clearly, more work is necessary to find effective agents that will protect against CIPN and allow for the antitumor activity of neurotoxic chemotherapeutic agents.

\section{Acknowledgments}

This work was supported in part by grants NCI-CA37404 (to C.L. Loprinzi and L.E. Ta) and NIDCR-DE020868 (to L.E. Ta), and by a Karl-Erivan Haub Family Career Development Award in Cancer Research (to L.E. Ta). We apologize to the many authors whose work has not been cited due to space limitations.

Address correspondence to: Charles L. Loprinzi, Mayo Clinic, 200 First Street, SW, Rochester, Minnesota 55905, USA. Phone: 507.284.4849; Fax: 507.284.1803; E-mail: cloprinzi@mayo.edu.

1. Tofthagen C, Donovan KA, Morgan MA, Shibata D, Yeh Y. Oxaliplatin-induced peripheral neuropathy's effects on health-related quality of life of colorectal cancer survivors. Support Care Cancer. 2013; 21(12):3307-3313.

2. Carozzi VA, Marmiroli P, Cavaletti G. The role of oxidative stress and anti-oxidant treatment in platinum-induced peripheral neurotoxicity. Curr Cancer Drug Targets. 2010;10(7):670-682.

3. Jiang Y, Guo C, Vasko MR, Kelley MR. Implications of apurinic/apyrimidinic endonuclease in reactive oxygen signaling response after cisplatin treatment of dorsal root ganglion neurons. Cancer Res. 2008; 68(15):6425-6434.
4. Miyamoto T, Dubin AE, Petrus MJ, Patapoutian A. TRPV1 and TRPA1 mediate peripheral nitric oxide-induced nociception in mice. PloS One. 2009; 4(10):e7596.

5. Nassini R, et al. Oxaliplatin elicits mechanical and cold allodynia in rodents via TRPA1 receptor stimulation. Pain. 2011;152(7):1621-1631.

6. Ta LE, Bieber AJ, Carlton SM, Loprinzi, CL, Low PA, Windebank AJ. Transient Receptor Potential Vanilloid 1 is essential for cisplatin-induced heat hyperalgesia in mice. Mol Pain. 2010;6:15

7. Xiao WH, Bennett GJ. Effects of mitochondrial poisons on the neuropathic pain produced by the chemotherapeutic agents, paclitaxel and oxaliplatin. Pain. 2012;153(3):704-709.

8. Ta LE,et al. A novel and selective poly (ADP-ribose) polymerase inhibitor ameliorates chemotherapy-induced painful neuropathy. PloS One. 2013; 8(1):e54161.

9. Flatters SJ, Xiao WH,Bennett GJ. Acetyl-L-carnitine prevents and reduces paclitaxel-induced painful peripheral neuropathy. Neurosci Lett. 2006; 397(3):219-223.

10. Boyette-Davis J,Dougherty PM. Protection against oxaliplatin-induced mechanical hyperalgesia and intraepidermal nerve fiber loss by minocycline. Exp Neurol. 2011;229(2):353-357.

11. Cavaletti G, Minoia C, Schieppati M, Tredici G. Protective effects of glutathione on cisplatin neurotoxicity in rats. Int J Radiat Oncol Biol Phys. 1994; 29(4):771-776

12. Bianchi R et al. Protective effect of erythropoietin and its carbamylated derivative in experimental Cisplatin peripheral neurotoxicity. Clin Cancer Res. 2006;12(8):2607-2612.

13. Ushio $S$, et al. Goshajinkigan reduces oxaliplatin-induced peripheral neuropathy without affecting anti-tumour efficacy in rodents. EurJ Cancer. 2012; 48(9):1407-1413.

14. Coriat R, et al. Treatment of oxaliplatin-induced peripheral neuropathy by intravenous mangafodipir. J Clin Invest. 2014;124(1):262-272.

15. Gilardini A, et al. Myelin structure is unaltered in chemotherapy-induced peripheral neuropathy. Neurotoxicology. 2012;33(1):1-7.

16. Argyriou AA, Kalofonos HP. Vitamin E for preventing chemotherapy-induced peripheral neuropathy. Support Care Cancer. 2011;19(5):725-726.

17. Kottschade L, Sloan J, Loprinzi C. Second response to the letter to the editor referencing the manuscript the "use of vitamin E for the prevention of chemotherapy-induced peripheral neuropathy: results of a randomized phase III clinical trial". Support Care Cancer. 2013;21(1):3-4.

18. Pace A, Galie E, Koudriavtseva T. Neuroprotective strategies in the prevention of chemotherapy-induced neuropathies. Support Care Cancer. 2013; 21(1):1-2.

19. Argyriou AA, et al. Preventing paclitaxel-induced peripheral neuropathy: a phase II trial of vitamin E supplementation. J Pain Symptom Manage. 2006; 32(3):237-244.

20. Argyriou AA, et al. A randomized controlled trial evaluating the efficacy and safety of vitamin E supplementation for protection against cisplatin-induced peripheral neuropathy: final results. Support Care Cancer. 2006;14(11):1134-1140.

21. Pace A, et al. Neuroprotective effect of vitamin $E$ supplementation in patients treated with cisplatin chemotherapy. J Clin Oncol. 2003;21(5):927-931.

22. Pace A, et al. Vitamin E neuroprotection for cisplatin neuropathy: a randomized, placebo-controlled trial. Neurology. 2010;74(9):762-766.

23. Kottschade LA, et al. The use of vitamin $\mathrm{E}$ for the prevention of chemotherapy-induced peripheral neuropathy: results of a randomized phase III clinical trial. Support Care Cancer. 2011; 19(11):1769-1777. 
24. Gamelin L. et al. Prevention of oxaliplatin-related neurotoxicity by calcium and magnesium infusions: a retrospective study of 161 patients receiving oxaliplatin combined with 5-Fluorouracil and leucovorin for advanced colorectal cancer. Clin Cancer Res. 2004;10(12 pt 1):4055-4061.

25. Grothey A, et al. Intravenous calcium and magnesium for oxaliplatin-induced sensory neurotoxicity in adjuvant colon cancer: NCCTG N04C7. J Clin Oncol. 2011;29(4):421-427.

26. Gamelin L, et al. Oxaliplatin-related neurotoxicity: interest of calcium-magnesium infusion and no impact on its efficacy. J Clin Oncol. 2008; 26(7):1188-1189.

27. Hochster HS, Grothey A, Childs BH. Use of calcium and magnesium salts to reduce oxaliplatin-related neurotoxicity. J Clin Oncol. 2007;25(25):4028-4029.

28. Loprinzi C, et al. Phase III randomized, placebo (PL)-controlled, double-blind study of intravenous calcium/magnesium (CaMg) to prevent oxaliplatin-induced sensory neurotoxicity (sNT), N08CB: An alliance for clinical trials in oncology study. J Clin Oncol. In press.

29. van der Hoop RG, et al Prevention of cisplatin neurotoxicity with an $\mathrm{ACTH}(4-9)$ analogue in patients with ovarian cancer. N Engl J Med. 1990;322(2):89-94.

30. Hovestadt A, van der Burg ME, Verbiest HB, van Putten WL, Vecht CJ. The course of neuropa- thy after cessation of cisplatin treatment, combined with Org 2766 or placebo. J Neurol. 1992; 239(3):143-146.

31. van Gerven JM, et al. The effects of an ACTH (4-9) analogue on development of cisplatin neuropathy in testicular cancer: a randomized trial. J Neurol. 1994;241(7):432-435.

32. van Kooten B, et al. A pilot study on the influence of a corticotropin (4-9) analogue on Vinca alkaloid-induced neuropathy. Arch Neurol. 1992; 49(10):1027-1031.

33. Koeppen S, et al. Lack of neuroprotection by an ACTH (4-9) analogue. A randomized trial in patients treated with vincristine for Hodgkin's or non-Hodgkin's lymphoma. J Cancer Res Clin Oncol. 2004;130(3):153-160.

34. Roberts JA, Jenison EL, Kim K, Clarke-Pearson D, Langleben A. A randomized, multicenter, double-blind, placebo-controlled, dose-finding study of ORG 2766 in the prevention or delay of cisplatin-induced neuropathies in women with ovarian cancer. Gynecol Oncol. 1997;67(2):172-177.

35. Cascinu S. et al. Neuroprotective effect of reduced glutathione on oxaliplatin-based chemotherapy in advanced colorectal cancer: a randomized, double-blind, placebo-controlled trial. J Clin Oncol. 2002;20(16):3478-3483.

36. Milla P, Airoldi M, Weber G, Drescher A, Jaehde U,
Cattel L. Administration of reduced glutathione in FOLFOX4 adjuvant treatment for colorectal cancer: effect on oxaliplatin pharmacokinetics, Pt-DNA adduct formation, and neurotoxicity. Anticancer Drugs. 2009;20(5):396-402.

37. Smyth JF, et al. Glutathione reduces the toxicity and improves quality of life of women diagnosed with ovarian cancer treated with cisplatin: results of a double-blind, randomised trial. Ann Oncol. 1997; 8(6):569-573

38. Bianchi, G. et al. Symptomatic and neurophysiological responses of paclitaxel- or cisplatin-induced neuropathy to oral acetyl-L-carnitine. EurJ Cancer. 2005; 41(12):1746-1750.

39. Hershman DL, et al. Randomized double-blind placebo-controlled trial of acetyl-L-carnitine for the prevention of taxane-induced neuropathy in women undergoing adjuvant breast cancer therapy. J Clin Oncol. 2013;31(20)2627-2633.

40. André T, et al. The IDEA (International Duration Evaluation of Adjuvant Chemotherapy) Collaboration: Prospective Combined Analysis of Phase III Trials Investigating Duration of Adjuvant Therapy with the FOLFOX (FOLFOX4 or Modified FOLFOX6) or XELOX (3 versus 6 months) Regimen for Patients with Stage III Colon Cancer: Trial Design and Current Status. Curr Colorectal Cancer Rep. 2013; 9:261-269.

\title{
Hostile takeover: fungal protein promotes host cell invasion
}

\author{
J. Andrew Alspaugh
}

Department of Medicine, Department of Molecular Genetics and Microbiology, Duke University School of Medicine, Durham, North Carolina, USA.

\begin{abstract}
The ability to suppress the immune system has lead to great advances in transplant technology and treatment of autoimmune diseases. Unfortunately, the immunosuppression of these patients has led to the rise of opportunistic infections by organisms that are recalcitrant to current prophylactic strategies. One such example is the increase of mucormycosis, an invasive infection caused by filamentous fungi of the order Mucorales. In this issue of the JCI, Gebremariam and colleagues determined that spore coat homolog $(\mathrm{CotH})$ proteins are required for angioinvasion and that these proteins are unique to Mucorales. Their findings provide a potential therapeutic target for prevention and treatment of mucormycosis.
\end{abstract}

\section{The rise of mucormycosis}

Recent medical advances have made remarkable progress in treating previously refractory conditions. More aggressive and targeted cancer chemotherapies have vastly improved outcomes for many malignancies. Inhibition of TNF- $\alpha$ activity now affords better control of various autoimmune disorders. Moreover, advances in solid organ transplantation have dramatically improved the lives of many patients

Conflict of interest: The author has declared that no conflict of interest exists.

Citation for this article: J Clin Invest. 2014; 124(1):74-76. doi:10.1172/JCI73585. with organ failure syndromes. However, these revolutionary therapies considerably impair patient immunity.

Because of the increased infection risk in patients with highly immunocompromised states, clinicians have adopted concrete strategies for infectious disease prevention in many of these patient populations. Unfortunately, as our ability to suppress infections by the most common microbial pathogens has improved, other, less well-characterized infectious agents have begun to fill this clinical void. One very important example of this phenomenon is the increasing incidence of mucormycosis, an invasive infection caused by the Mucorales order of filamentous fungi $(1,2)$. Human pathogens in this fungal group include Rhizopus, Mucor, and Cunninghamella species.

The increased incidence of mucormycosis has been attributed to many factors, including the fact that Mucorales are much less susceptible to current antifungal agents than other fungal pathogens. Therefore, the use of standard antifungal drugs in prophylactic strategies is unlikely to successfully prevent this type of infection $(3,4)$. Mucormycosis occurs in association with a wide range of disorders. In addition to classical immunocompromised states, such as prolonged neutropenia and organ transplantation, conditions such as diabetic ketoacidosis (DKA), undernutrition, and iron chelation therapy also predispose patients to mucormycosis (2). Once established, invasive infections due to Mucorales frequently take an aggressive clinical course characterized by rapid tissue destruction. These infections are difficult to treat, requiring high-dose antifungal therapy and surgical debridement. Mortality in mucormycosis remains high, despite 